\title{
Hybrid inorganic-organic nano- and microcomposites based on silica sols and synthetic polyelectrolytes
}

\author{
S. E. Kudaibergenov ${ }^{1,2,3 *}$, G. S. Tatykhanova ${ }^{3}$, B. Zh. Arinov², S. K. Kozhakhmetov ${ }^{2}$, V. O. Aseyev ${ }^{4}$ \\ ${ }^{1}$ Institute of Polymer Materials and Technology, Almaty, 050004, Republic of Kazakhstan \\ 2JSC Scientific-Production Center 'Ulba', National Atomic Company 'Kazatomprom', Ust-Kamenogorsk, 070005, \\ Republic of Kazakhstan \\ ${ }^{3}$ Semipalatinsk State Shakarim University, Semipalatinsk, Republic of Kazakhstan \\ ${ }^{4}$ Helsinki University, Laboratory of Polymer Chemistry, Helsinki, Finland
}

Received 18 November 2007; accepted in revised form 25 December 2007

\begin{abstract}
Interaction between anionic (and cationic) colloidal particles of silica having the particles diameters 12 and $22 \mathrm{~nm}$ with synthetic cationic (and anionic) polyelectrolytes of various nature and structure was studied by potentiometric, conductimetric spectroturbidimetric and viscometric methods in aqueous solution. It was shown that the complexation of silica nanoparticles with linear polyelectrolytes leads to formation of mostly stoichiometric interpolyelectrolyte complexes (IPEC) which precipitate from aqueous solution. Casting of water-soluble IPEC followed by thermal treatment gives thin composite films insoluble in water while 'layer by layer' (LbL) deposition of polyelectrolyte components onto silica sols leads to formation of multilayered nano- and microcomposites. The possible mechanism of formation of LbL multilayers consisting of silica sol $\left(\mathrm{SiO}_{2}\right)$ 'cores' and polyethyeleneimine-polyacrylic acid (PEI-PAA) 'shells' was suggested. It was found that in diluted aqueous solution the radius of gyration, $R_{g}$ and hydrodynamic radius, $R_{h}{ }^{\text {mean }}$ of LbL particles are independent on LbL concentration and smaller than $100 \mathrm{~nm}$. The zeta potential values of LbL particles are arranged between -10 and $-30 \mathrm{mV}$. The average size of LbL particles estimated by scanning electron microscopy (SEM) is in the range of 200-500 nm. Thermal treatment of LbL multilayers followed by etching of $\left(\mathrm{SiO}_{2}\right)$ 'core' by $\mathrm{HF}$ leads to formation of a series of spherical nanocavities and blob-like microcavities.
\end{abstract}

Keywords: nanomaterials, silica nanoparticles, linear polyelectrolytes, interpolyelectrolyte complexes

\section{Introduction}

Hybrid inorganic-organic nanocomposites comprising inorganic nanoparticles and functional polymers are novel and unique class of nanomaterials that synergistically combine and enhance the best properties of inorganic and organic polymers [1-4]. The potential application fields of such hybrids can include specialty coatings, membranes, sensors, biomaterials, drug delivery systems, catalysts etc. The most common principle concepts of incorporation of inorganic nanoparticles into polymer matrix and the resulting properties of such materials have been reviewed by authors [5-7]. Classification of polymer/silicate nanocomposites based on synthetic techniques was suggested in [8]. Although titanium $[9,10]$ and aluminum [11] oxides have been utilized as the inorganic components interacting with functional polymers, the colloid silica due to easy preparation by sol-gel technology [12] is one of the promising inorganic polymeric materials that interacts with polyelectrolytes and forms, so called interpolyelectrolyte complexes (IPEC) - products of interaction of two oppositely charged polyelectrolytes [13-17]. Mech-

*Corresponding author, e-mail: ipmt-kau@usa.net

(C) BME-PT and GTE 
anisms of formation, structure and properties of IPECs based on synthetic polyelectrolytes have been thoroughly investigated by Bixler and Michaelis [18], Tsuchida and Abe [19], Kabanov [17], Zezin and Kabanov [20], Bekturov and Bimendina [21], Philipp et al. [22]. Polyelectrolyte complex formation between strong polyanion and cationic nanogels was studied by Ogawa et al. [23]. Recently [24] the multilayered nanomaterials were developed on the basis of hydrogen-bonded complexes of polyacrylic acid and methylcellulose.

Ultrathin membranes with participation of colloidal particles and cationic polyelectrolytes [25], layered double hydroxide and anionic polyelectrolytes [26], and colloidal biocatalysts consisting of polystyrene-based colloid particles coated with glucose oxidase and hoseradish peroxidase [27] as well as polyelectrolyte composite films [28] and nanotubes [29] were assembled via the LbL self-assembling. Hybrid ternary organic-inorganic films consisting of chitosan, poly(monomethyl)itaconate and silica nanoparticles were designed by Martinez et al. [30] and further the same authors [31] embedded the semiconductor $\mathrm{CdS}$ nanoparticles into organicinorganic hybrid by in situ technique. Thus the understanding of the basic principles of fabrication of composite inorganic-organic nano- and micromaterials where one component phase is nanoparticle is a challenging task [32].
The present communication deals with studying of the formation and characterization of hybrid inorganic-organic nano- and microcomposites as a result of interpolyelectrolyte complexation between silica nanoparticles and linear polyelectrolytes.

\section{Experimental}

\subsection{Materials}

Colloidal particles of silica are commercial products purchased from Ludox (Grace $\mathrm{GmbH} \& \mathrm{Co}$. $\mathrm{KG}$, Worms, Germany) were abbreviated as HS-40, AS-40 and CL in dependence of surface charge, nature of counterions, particle size and surface area. Table 1 represents some physico-chemical characteristics of anionic and cationic nanoparticles of silica.

Table 1. Physico-chemical characteristics of colloidal silica nanoparticles

\begin{tabular}{|l|c|c|c|}
\hline \multirow{2}{*}{\multicolumn{1}{|c|}{ Characteristics }} & \multicolumn{3}{|c|}{ Type of colloidal particles } \\
\cline { 2 - 4 } & HS-40 & AS-40 & CL \\
\hline Surface charge & - & - & + \\
\hline Counterion & $\mathrm{Na}^{+}$ & $\mathrm{NH}_{4}{ }^{+}$ & $\mathrm{Cl}^{-}$ \\
\hline $\mathrm{SiO}_{2}[\mathrm{wt} \%]$ & 40 & 40 & 30 \\
\hline $\mathrm{Na}_{2} \mathrm{O}[\mathrm{wt} \%]$ & 0.41 & $0.05-0.08$ & - \\
\hline Particles size $[\mathrm{nm}]$ & 12 & 22 & 12 \\
\hline Surface area $\left[\mathrm{m}^{2} / \mathrm{g}\right]$ & 240 & 140 & 240 \\
\hline $\mathrm{pH}\left(25^{\circ} \mathrm{C}\right)$ & 9.7 & 9.1 & 4.5 \\
\hline
\end{tabular}

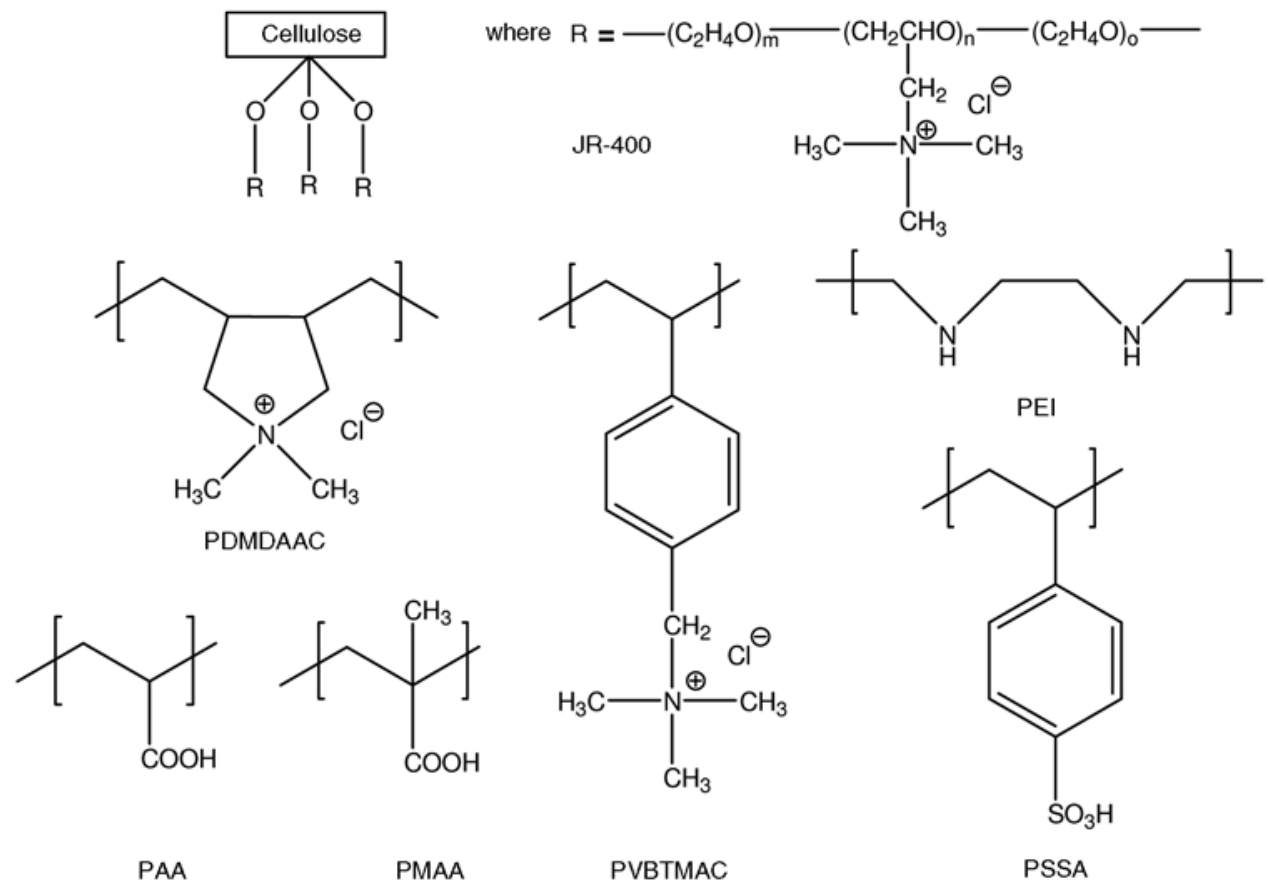

Figure 1. Structural formulas of linear polyelectrolytes 
Table 2. Some of physico-chemical characteristics of linear polyelectrolytes

\begin{tabular}{|l|c|c|c|c|c|c|c|}
\hline \multirow{2}{*}{\multicolumn{1}{c|}{ Characteristics }} & \multicolumn{7}{c|}{ Linear polyelectrolytes } \\
\cline { 2 - 9 } & JR-400 & PDMDAAC & PVBTMAC & PEI & PSSA & PAA & PMAA \\
\hline Surface charge & + & + & + & + & - & - & - \\
\hline Counterion & $\mathrm{Cl}^{-}$ & $\mathrm{Cl}^{-}$ & $\mathrm{Cl}^{-}$ & $\mathrm{OH}^{-}$ & $\mathrm{H}^{+}$ & $\mathrm{H}^{+}$ & $\mathrm{H}^{+}$ \\
\hline $\mathrm{M} \cdot 10^{-3}[$ Dalton] & $100-3000$ & 219 & 100 & 25 & 500 & 450 & 250 \\
\hline $\mathrm{pH}\left(25^{\circ} \mathrm{C}\right)$ & 5,66 & 4,90 & 5,48 & 8,37 & 8,01 & 3,00 & 3,40 \\
\hline
\end{tabular}

As linear cationic and anionic polyelectrolytes the quaternized ammonium salts of cellulose derivatives (JR-400) [33], poly-N,N-dimethyl-N,N-diallylammonium chloride (PDMDAAC), poly-Nvinylbenzyl-N,N,N-trimethylammonium chloride (PVBTMAC), polyethyleneimine (PEI), polystyrenesulfonic acid (PSSA), polyacrylic (PAA) and polymethacrylic (PMAA) acids purchased from Polysciences, Inc. (Warrington, PA, USA) were used. Structural formulas of water-soluble polyelectrolytes and some of their physico-chemical characteristics are represented in Figure 1 and Table 2. In addition to linear polyelectrolytes, microgel of PDMDAAC with average diameters of particles $1-3 \mu \mathrm{m}$ was used.

\subsection{Methods}

Potentiometric and conductimetric titrations were carried out with the help of combined $\mathrm{pH} / \mathrm{Conduc}$ tometer 'Mettler-Toledo MPC-227' (Schweiz, Switzerland) at room temperature. Turbidity of solutions was measured by photoelectrocolorimeter 'FEK-56' (Moscow, Russia) at $\lambda=340 \mathrm{~nm}$ and room temperature. Viscometric measurements were carried out on Ubbelohde viscometer at $298 \pm 0.1 \mathrm{~K}$. Optical microscope of LOMO (Saint-Petersburg, Russia) was used for observation of thin powders and multilayered films structures.

Dynamic and static light scattering experiments were conducted with a Brookhaven Instruments (NY, USA) BI-200SM goniometer, a BI-TurboCorr digital auto/crosscorrelator, and a BI-CrossCorr detector, including two BI-DS1 detectors. A Sapphire 488-100 CDRH laser from Coherent $\mathrm{GmbH}$ operating at the wavelength of $\lambda_{0}=488 \mathrm{~nm}$ (vertically polarized) and the power adjusting from 10 to $50 \mathrm{~mW}$ was a light source. The scattering angles were varied in the range of $\theta=30-150^{\circ}$. In the DLS experiments, pseudo cross-correlation functions of the scattered light intensity, $G_{2}(t)$, were collected with the self-beating method [34].
The time average intensity of the scattered light, $I_{\theta}$, was recorded simultaneously. The intensities measured in counts of photons per second, cps, were normalised with respect to the Rayleigh ratio of toluene. The temperature of the samples was controlled by means of a Lauda RC 6C thermostated water bath. For light scattering experiments the selected concentrations of LbL samples were $C_{p}=0.121,0.605$, and $12.1 \mathrm{mg} / \mathrm{ml}$. Aqueous solutions were passed through the hydrophilic MillexHV $0.45 \mu \mathrm{m}$ pore size and $13 \mathrm{~mm}$ in diameter filters prior to measurements to remove dust particles.

Zeta-potential measurements were performed with a Nano-ZS Zetasizer ZEN3600 from Malvern Instruments (Malvern, UK) equipped with $4 \mathrm{~mW}$ $\mathrm{He}-\mathrm{Ne}$ laser operating at $\lambda_{0}=633 \mathrm{~nm}$. Nano-ZS Zetasizer is based on the back scattering data detection $\left(\theta=173^{\circ}\right)$. M3-PALS technique and Smoluchowski analysis is used to measure the zeta-potential. Size \& Zeta-potential-DTS1060 folded capillary cell was employed for the simultaneous size and zeta-potential measurements. Temperature was stabilized with a Peltier temperature control.

SEM pictures were obtained for freeze-dried LbL samples using Hitachi S-4800 (Tokyo, Japan) instrument. Freeze-drying solution was deposited on a grid for SEM and quickly frozen under liquid nitrogen. The grid then was placed in vacuum to remove water.

\subsection{Preparation of thin powders, composite films and layer-by-layer compositions of IPEC}

Bidistilled water was used for preparation of colloidal particles and polyelectrolyte solutions. Aqueous solutions of polyelectrolytes $(0.01 \mathrm{~mol} / \mathrm{l})$ were added dropwise to the aqueous solution of colloidal silica $(0.01 \mathrm{~mol} / \mathrm{l})$ under stirring. The precipitated IPEC particles were separated from supernatant by preparative centrifuge 'T-62' (Warsaw, 


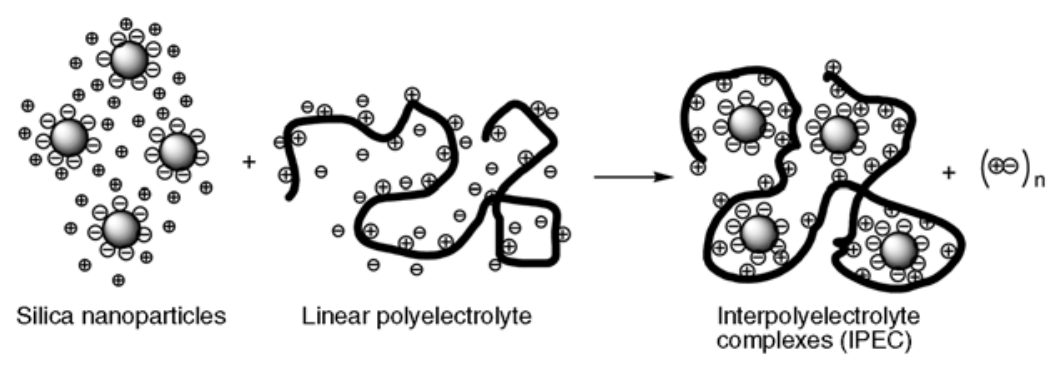

Figure 2. Scheme of IPEC formation between silica sols and linear polyelectrolytes

Poland) at $5000 \mathrm{rpm}$. They were then washed with acetone several times. After drying for one day in vacuum oven, the IPEC particles were obtained in the form of thin powder.

Since the IPEC obtained by mixing of aqueous solutions of HS-40 and JR-400 preserved in aqueous solution, by casting the equimolar mixture of HS-40 and JR-400 onto Teflon surface the thin films were fabricated. After thermal treatment of thin films at $100^{\circ} \mathrm{C}$ for one day they became insoluble but swellable in water. The LbL assembly process developed for planar solid substrates was adapted for silica sols. At first the dilute aqueous solutions of silica sols, PEI and PAA with concentration of $\mathrm{C}=10^{-3} \mathrm{~mol} / \mathrm{l}$ were prepared. To $1 \mathrm{ml}$ of AS-40 $1 \mathrm{ml}$ of PEI was added under stirring during $5 \mathrm{~min}$. Then $1 \mathrm{~mL}$ of PAA was added to $2 \mathrm{ml}$ mixture of AS-40 and PEI. Sequential addition of PAA and PEI to AS-40/PEI was continuously repeated 10 times under constant stirring time $5 \mathrm{~min}$ for each stage. The obtained solution consisting of $\mathrm{SiO}_{2}$ 'core' and PEI-PAA 'shell' was abbreviated as $\left(\mathrm{SiO}_{2}\right) /(\mathrm{PEI}-\mathrm{PAA})_{10}$ (where 10 correspond to number of PEI-PAA multilayers). One drop of opalescent solution of $\left(\mathrm{SiO}_{2}\right) /(\mathrm{PEI}-\mathrm{PAA})_{10}$ was deposited onto the glass surface, dried in air and then heated in oven at $100^{\circ} \mathrm{C}$ during $6 \mathrm{~h}$. Etching of $\mathrm{SiO}_{2}$ from $\left(\mathrm{SiO}_{2}\right) /(\mathrm{PEI}-\mathrm{PAA})_{10}$ compositions was provided by dipping the glass surface into $0.01 \mathrm{~mol} / \mathrm{l} \mathrm{HF}$ during $1 \mathrm{~min}$ and rinsing with bidistilled water. This procedure was repeated the required times. Finally the multilayered coatings were dried in air.

\section{Results and discussion}

According to [13-16] the mechanism of interaction of silicate sols with linear polyelectrolytes can be considered as cooperative adsorption of negatively charged $\mathrm{SiO}_{2}$ nanoparticles onto positively charged polyelectrolytes (Figure 2). The addition of a poly-

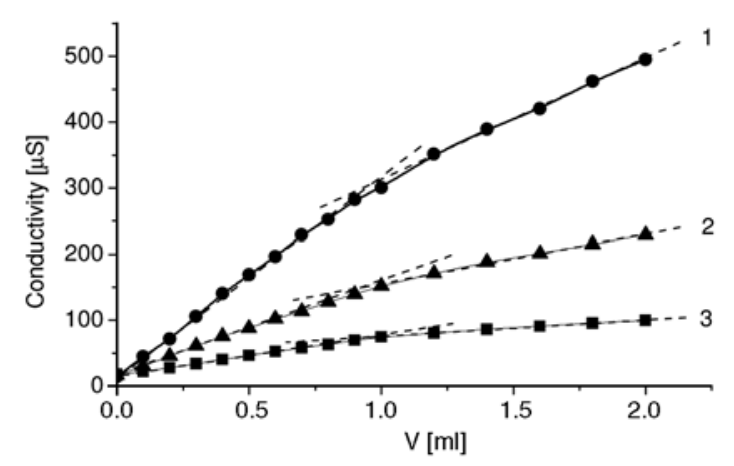

Figure 3. Conductimetric titration curves of colloidal dispersions of CL (1), AS-40 (2) and HS-40 (3) by PSSA (1), JR-400 (2) and PDMDAAC (3)

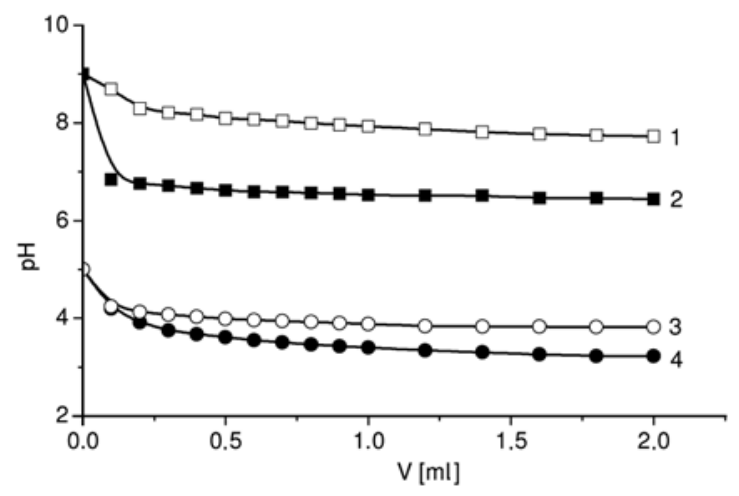

Figure. 4. Potentiometric titration curves of $\operatorname{HS}-40(1,2)$ and CL $(3,4)$ by PDMDAAC (1), JR-400 (2), PAA (3) and PMAA (4)

cation to a solution of a silicate ions results in precipitation of IPEC.

Figures 3 and 4 illustrate the conductometric and potentiometric titration curves of silicate sols by linear polyelectrolytes. Compositions of IPEC found from the inflection points are equal or close to equimolar ratio of interacting components (Table 3). Increasing of the optical density and viscosity of systems indicates an aggregation of IPEC particles (Figure 5).

Photos of colloidal silica, polyelectrolyte solution, precipitated IPEC, and IPEC powders are shown in Figure 6. 

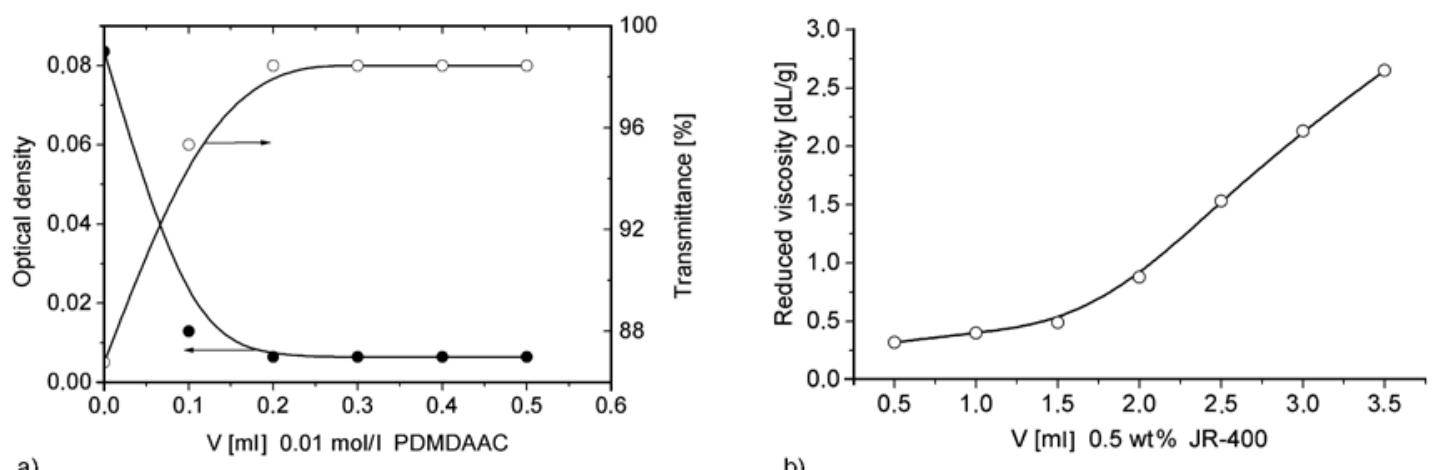

a)

b)

Figure 5. Spectroturbidimetric (a) and viscometric (b) titration curves of colloidal silica AS-40 by PVBTMAC (a) and JR-400 (b)

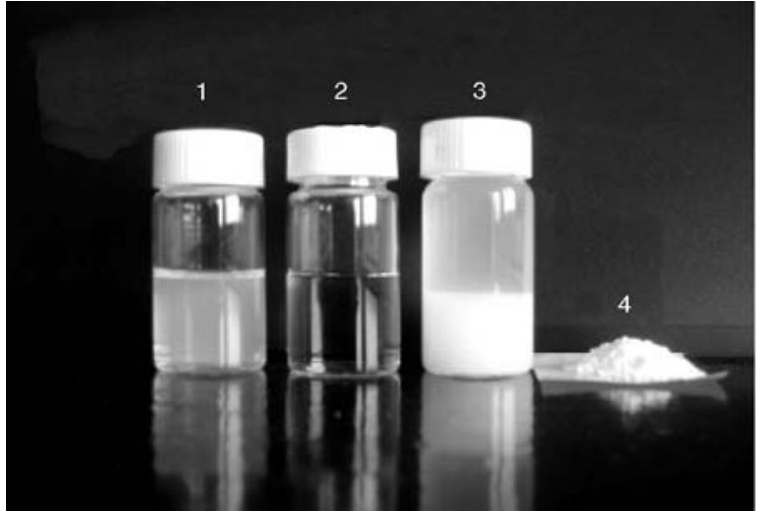

Figure 6. Photos of colloidal silica AS-40 (1), aqueous solution of JR-400 (2), precipitated IPEC (3), and IPEC thin powders (4)

Table 3. Compositions of IPEC determined from the inflection points of conductimetric and potentiometric titration curves

\begin{tabular}{|l|c|c|c|}
\hline \multirow{2}{*}{\multicolumn{1}{|c|}{ Polyelectrolytes }} & \multicolumn{3}{|c|}{ Composition of IPEC [mol/mol] } \\
\cline { 2 - 4 } & AS-40 & HS-40 & CL \\
\hline JR-400 & $1: 1.15$ & $1: 1$ & - \\
\hline PDMDAAC & $1: 1.04$ & $1: 1.33$ & - \\
\hline PVBTMAC & $1: 1.12$ & $1: 1$ & - \\
\hline PEI & $1: 1.05$ & $1: 1$ & - \\
\hline PSSA & - & - & $1: 1.2$ \\
\hline PAA & - & - & $1: 1.1$ \\
\hline PMAA & - & - & $1: 1$ \\
\hline PDMDAAC microgel & $1: 1$ & $1: 1$ & - \\
\hline
\end{tabular}

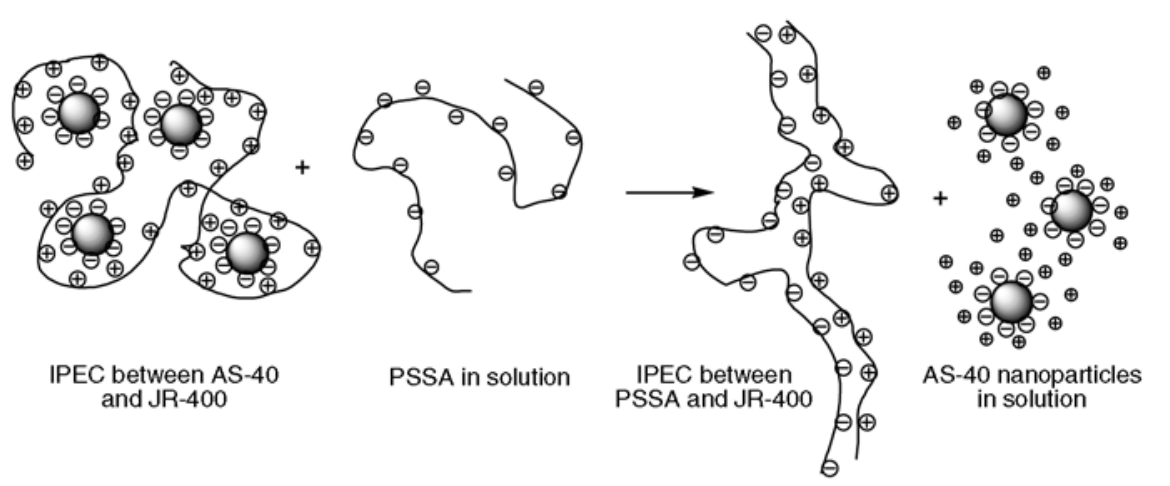

Figure 7. Release of silica sols from the IPEC as a result of competition reaction between IPEC and PSSA
Competition interpolyelectrolyte reactions between IPEC and strong polyelectrolytes are of great interest [17]. It is known that polyanions such as polyethylenesulfonate and polystyrenesulfonate are able to replace polycarboxylates, for instance PAA and PMAA, from the IPEC containing quaternized ammonium moieties as cationic polyelectrolytes. The driving force of competition reaction is specific, in addition to Coulomb forces, binding of sulfonate to quaternized ammonium groups. In this connection one can suppose that addition of aqueous solution of PSSA to IPEC based on AS-40 and JR-400 will lead to replacement of silica sols from the IPEC particles and formation of new IPEC between PSSA and JR-400. This is confirmed by conductometric titration of IPEC particles by aqueous solution of PSSA. The formation bigger flocs and the presence of inflection point on conductimetric curves at a molar ratio of [PSSA]:[JR-400] = $1: 1$ that coincides well with conductometric titration curves of JR-400 by PSSA and the appearance of $\mathrm{SiO}_{2}$ particles in supernatant confirms the release of silica sols from the initial IPEC. Interpolyelectrolyte reaction of replacement at which 
Table 4. Dimensions of the $\left(\mathrm{SiO}_{2}\right) /(\mathrm{PEI}-\mathrm{PAA})_{10} \mathrm{LbL}$ complex particles in water

\begin{tabular}{|c|c|c|c|c|c|}
\hline \multirow{2}{*}{$\begin{array}{c}\mathbf{L b L} \\
\mathbf{C}_{\mathbf{p}}[\mathbf{m g} / \mathbf{m l}]\end{array}$} & \multicolumn{2}{|c|}{ Zimm } & \multicolumn{2}{|c|}{ Guinier } & CONTIN \\
\cline { 2 - 6 } & $\mathbf{R}_{\mathbf{g}}{ }^{\mathbf{Z}}[\mathbf{n m}]$ & $\mathbf{R}_{\mathbf{g}}{ }^{\mathbf{Z}} / \mathbf{R}_{\mathbf{h}}{ }^{\text {'ean }}$ & $\mathbf{R}_{\mathbf{g}}{ }^{\mathbf{G}}[\mathbf{n m}]$ & $\mathbf{R}_{\mathbf{g}}{ }^{\mathbf{G}} / \mathbf{R}_{\mathbf{h}}{ }^{\text {mean }}$ & $\mathbf{R}_{\mathbf{h}}{ }^{\text {mean }}[\mathbf{n m}]$ \\
\hline $\mathrm{Cp} \rightarrow 0$ & 72.4 & 0.76 & 88.8 & 0.92 & 96.4 \\
\hline 0.121 & 70.7 & 0.74 & 82.9 & 0.87 & 95.7 \\
\hline 0.605 & 72.2 & 0.73 & 83.1 & 0.84 & 99.3 \\
\hline 12.1 & 74.5 & 0.81 & 89.6 & 0.97 & 92.4 \\
\hline
\end{tabular}

$\mathrm{SiO}_{2}$ particles are displaced by PSSA can schematically be represented by the following Figure 7 .

Thus IPEC formation between silica sols and cationic polyelectrolytes and interpolyelectrolyte competition reactions with participation of strong polyelectrolytes can serve as preparative and technological basis for extraction and purification of $\mathrm{SiO}_{2}$ nanoparticles.

The data of light scattering experiments for $\mathrm{LbL}$ consisting of $\left(\mathrm{SiO}_{2}\right) /(\mathrm{PEI}-\mathrm{PAA})_{10}$ are collected in Table 4. The main conclusion from these results is that the radius of gyration, $R_{g}$ and hydrodynamic radius, $R_{h}{ }^{\text {mean }}$ of LbL particles calculated by using of Zimm and Guinier methods and of an inverse Laplace program CONTIN are independent on LbL concentration and smaller than $100 \mathrm{~nm}$.

Following the LbL preparation procedure, the most expected conformation of (PEI-PAA) 10 shell layer is a swollen sphere (or a thick-walled sphere). The $R_{g} / R_{h}$ ratios listed in Table 4 support this model. The Zimm's method is typically used for structures, density of which increases towards the centre of gravity, e.g. a polymer coil. In this respect the Guinier's method should give more reliable value of $R_{g}$. We tried to estimate the geometrical parameters of such a thick-walled sphere. In this model the outer radius was taken as $R_{1}=R_{h}^{\text {mean }}=96.4 \mathrm{~nm}$, $R_{g}=R_{g}{ }^{G}=88.8 \mathrm{~nm}$, and the inner radius $R_{2}$ calculated from the Equation (1):

$$
R_{g}^{2}=\frac{3}{5} \cdot \frac{R_{1}^{5}-R_{2}^{5}}{R_{1}^{3}-R_{2}^{3}}
$$

was equal to $80.0 \mathrm{~nm}$.

It should be taken into account that measuring of the molar mass and $R_{g}$ of $\left(\mathrm{SiO}_{2}\right) /(\mathrm{PEI}-\mathrm{PAA})_{10}$ by light scattering methods is not a straightforward task due to the difference in the refractive indexes of the polymers [35]. In this respect, the measured value of $R_{g}$ might be somewhat underestimated, meaning that the actual wall thickness of the sphere is smaller. Feng et al. [36] fabricated hollow microcapsules having shells of polyelectrolyte complexes by polymerization of 4-vinylpyridine hydrochloride (VPy) in a solution containing surface-modified silica particles and poly(sodium 4styrenesulfonate) (NaPSS), and followed by a core removal with HF. The continuous shells with a thickness of 5-15 $\mathrm{nm}$ on the silica particles were created by covalent linking of the NaPSS-PVPy complexes.

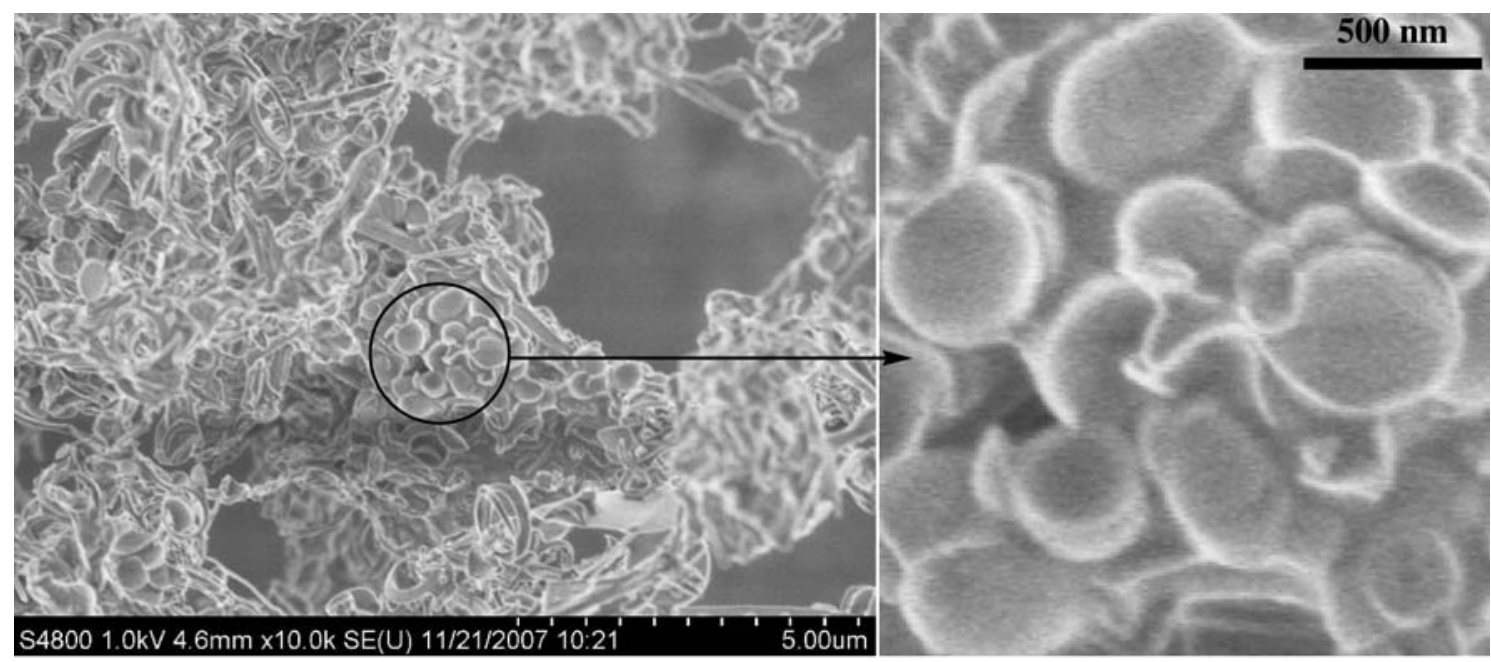

Figure 8. SEM picture of freeze-dried LbL particles of $\left(\mathrm{SiO}_{2}\right) /(\mathrm{PEI}-\mathrm{PAA})_{10}$. Arrow shows the enlarged area of circle 


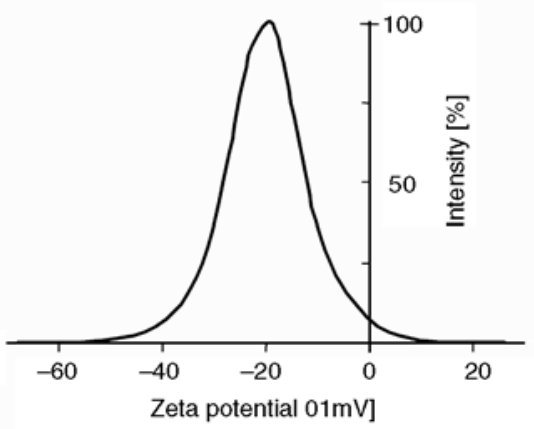

Figure 9. Zeta-potential measured for $\mathrm{LbL}$ of $\left(\mathrm{SiO}_{2}\right) /(\mathrm{PEI}-$ PAA) ${ }_{10}$ with $C_{p}=0.121 \mathrm{mg} / \mathrm{ml}$

SEM picture of freeze-dried $\left(\mathrm{SiO}_{2}\right) /(\mathrm{PEI}-\mathrm{PAA})_{10}$ particles prepared from $C_{p}=12.1 \mathrm{mg} / \mathrm{ml}$ is depicted in Figure 8. In spite of the fact that during the freeze-drying process the ice crystals can damage the outer shells, the clones of ball-like particles are observed. The average size of such particles is in the range of $200-500 \mathrm{~nm}$.

The zeta-potential of $\left(\mathrm{SiO}_{2}\right) /(\mathrm{PEI}-\mathrm{PAA})_{10}$ is shown in Figure 9. The mean peak value of the zeta-potential distributions fluctuates between -10 and $-30 \mathrm{mV}$ depending on the $\mathrm{LbL}$ concentration, which one could in principle be expected for saltfree aqueous solutions. The zeta-potential values and their sign agree well with the procedure of the complex preparation: the outer layer of the (PEIPAA) 10 complex consists of PAA.

Microphotographs of thermally treated $\left(\mathrm{SiO}_{2}\right) /$ (PEI-PAA) 10 powder before and after etching of $\mathrm{SiO}_{2}$ by $\mathrm{HF}$ are shown in Figure 10. Separate hollow spherical particles with different sizes and aggregated blob-like hollow particles bridged each other via hollow tubes are good seen. The mechanism of formation of LbL multilayers is probably multistage process (Figure 11). At first PEI chains may wrap one or several $\mathrm{SiO}_{2}$ nanoparticles (Figure 11a). The added PAA chains are deposited onto $\mathrm{SiO}_{2}$-PEI particles via electrostatic interactions (Figure 11b). Sequential adsorption of PEI and PAA to a single $\mathrm{SiO}_{2}$ and/or aggregated $\mathrm{SiO}_{2}$ nanoparticles can produce a spherical and/or cylindrical $\left(\mathrm{SiO}_{2}\right) /(\text { PEI-PAA })_{10}$ multilayers (Figure $11 \mathrm{c}, \mathrm{d})$. Here the dangled parts of PEI and PAA can participate in formation of IPEC particles and bridge the aggregated LbL particles (Figure 11d).

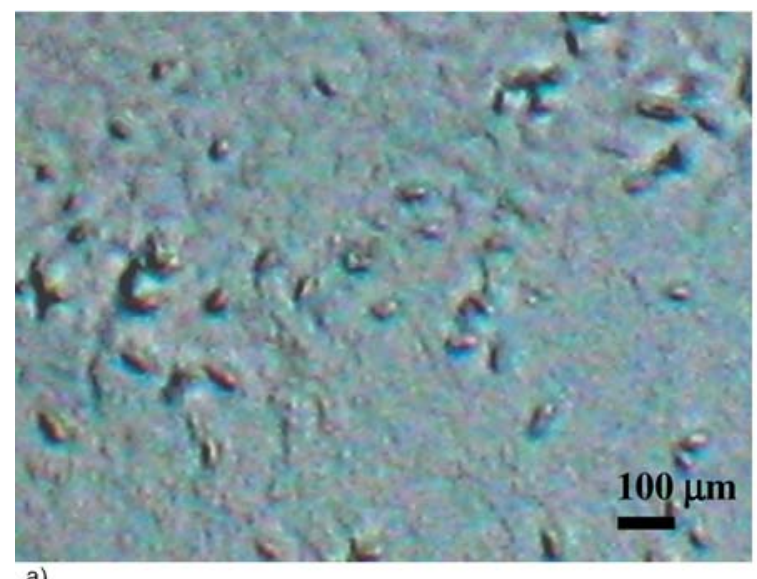

a)
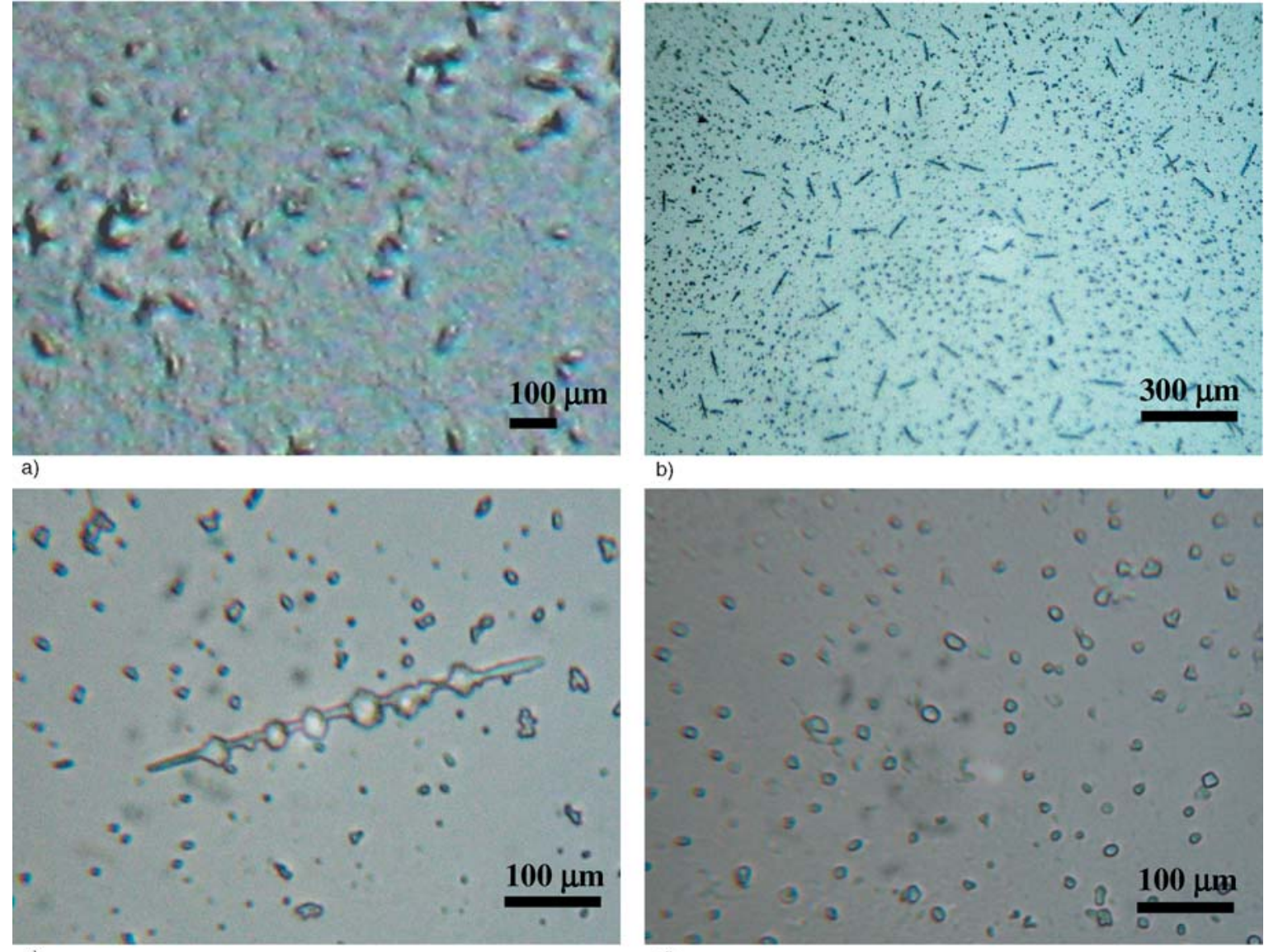

b)

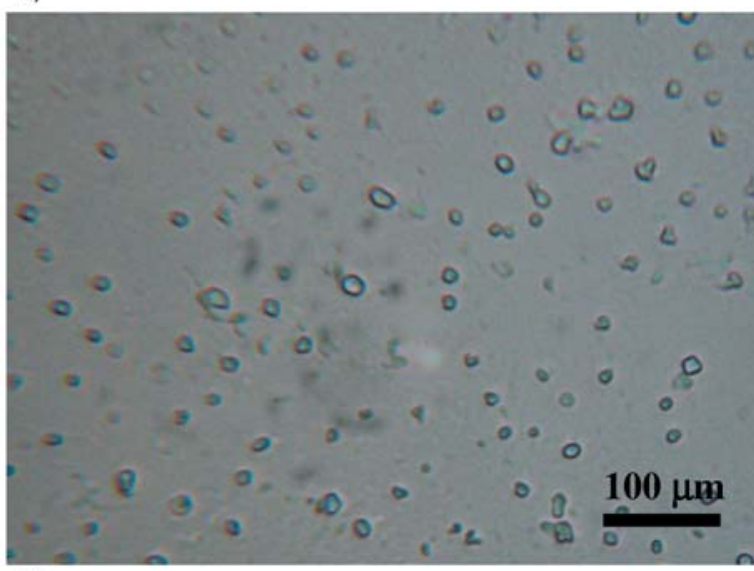

c)

d)

Figure 10. Microphotographs of $\left(\mathrm{SiO}_{2}\right) /(\mathrm{PEI}-\mathrm{PAA})_{10}$ after thermal treatment (a) and after etching by $\mathrm{HF}(\mathrm{b}, \mathrm{c}, \mathrm{d})$ 
a)

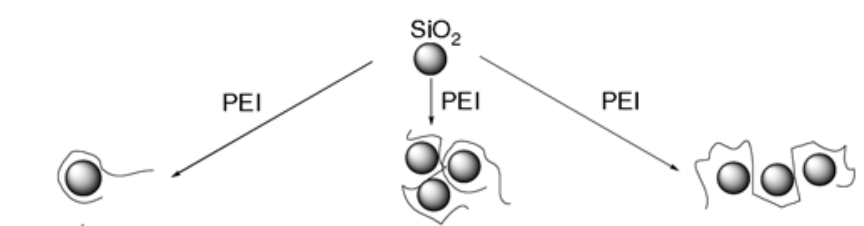

b)
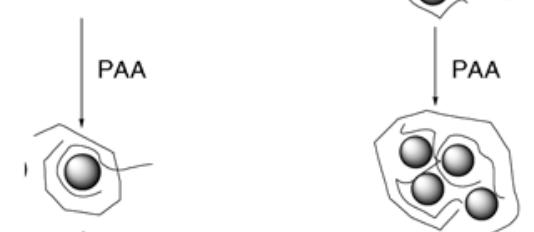

PEI

c)

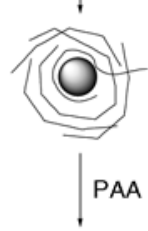

d)
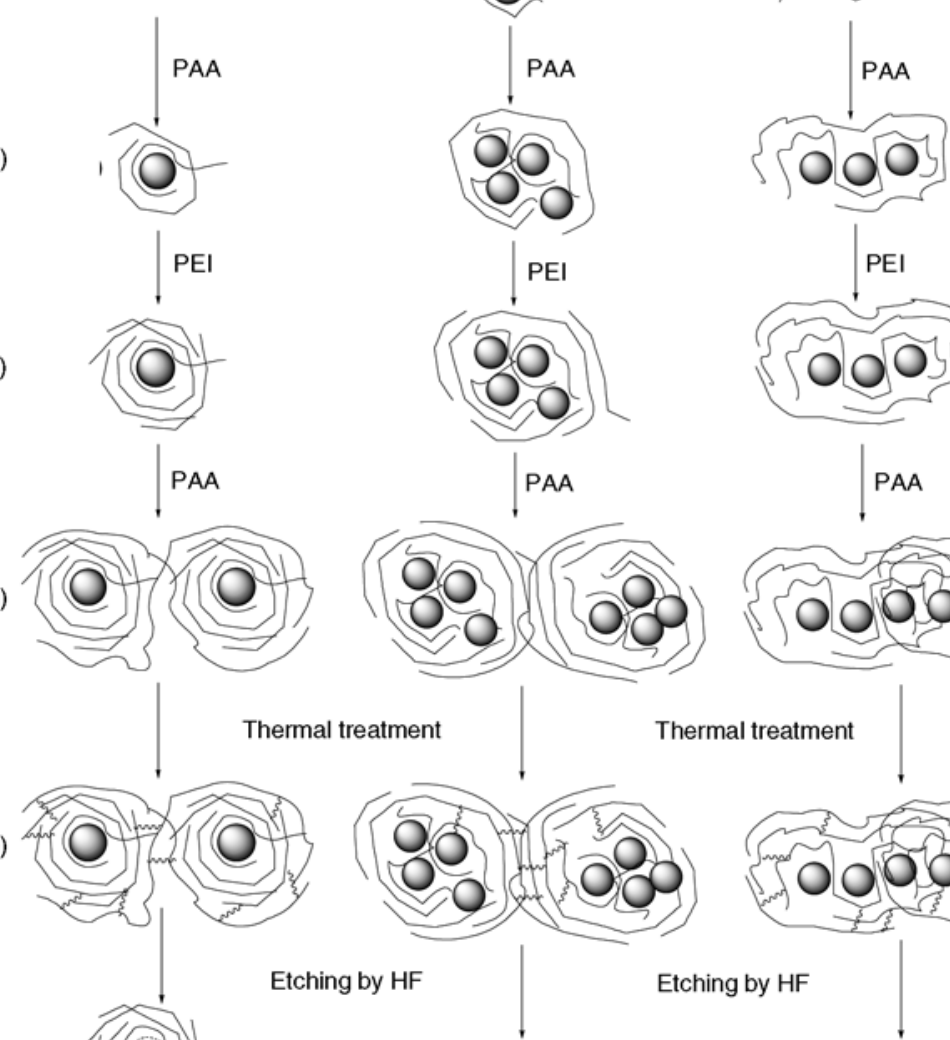

Thermal treatment
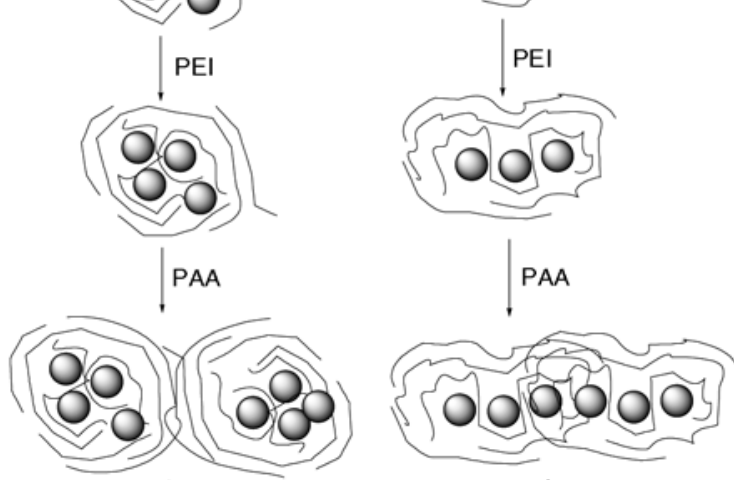

Thermal treatment
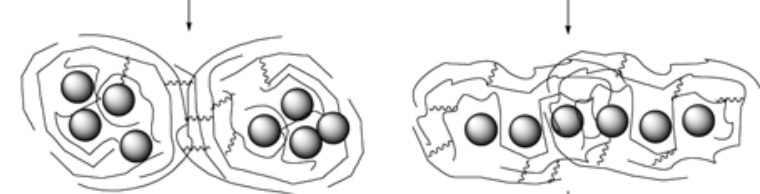

f)
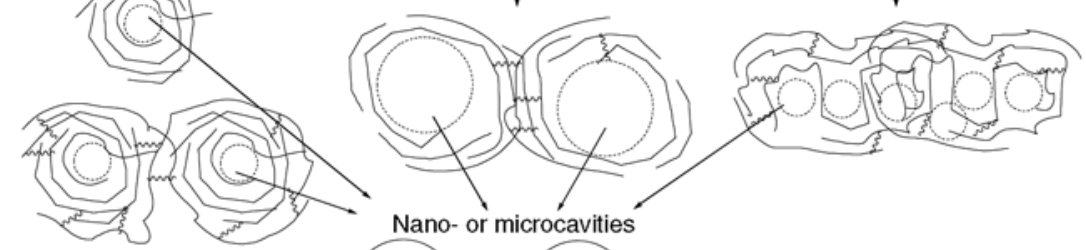

g)
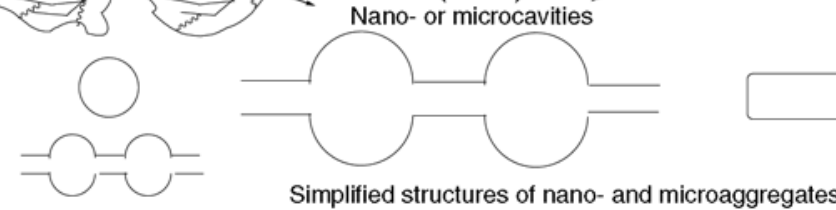

Figure 11. LbL multilayers assembled via interpolyelectrolyte reactions between silica sols and linear polyelectrolytes

The further thermal treatment of $\left(\mathrm{SiO}_{2}\right) /(\mathrm{PEI}-$ PAA) 10 multilayers or aggregates converts the ionic bonds $-\mathrm{NH}_{3}{ }^{+}{ }^{-} \mathrm{OOC}-$ on 'shell' layers to covalent amide bonds $-\mathrm{NH}-\mathrm{OC}-$ analogous to amidization of PEI-PAA interpolyelectrolyte complexes (Figure 11e) [37]. Etching of $\mathrm{SiO}_{2}$ 'cores' from $\mathrm{LbL}$ multilayers leads to formation of nano- and microcavities, sizes of which corresponds to single or aggregated $\mathrm{SiO}_{2}$ particles (Figure 11f). The simplified nano- and microparticles of $\left(\mathrm{SiO}_{2}\right) /(\mathrm{PEI}-$ PAA $)_{10}$ can be represented as single or aggregated nanocavities, blob-like microparticles and even nano- and microturbes (Figure 11g). In the course of LbL multilayers the growth of a various combinations of nano- and microparticles leading to different nano- and microstructures are possible.

\section{Conclusions}

Nano- and microsized hybrid inorganic-organic composites were assembled between colloidal silica sols and synthetic polyelectrolytes as a result of interpolyelectrolyte reactions. The composition of precipitated interpolyelectrolyte complex (IPEC) 
particles is mostly stoichiometric. Preparation of thin IPEC powders and composite films is demonstrated. Competition interpolyelectrolyte reactions between IPEC and strong polyelectrolytes, in particular polystyrenesulfonic acid (PSSA), can have technological importance for extraction and purification of $\mathrm{SiO}_{2}$ nanoparticles. The probable mechanism of formation of 'layer-by-layer' (LbL) multilayers is suggested. The LbL technique followed by thermal treatment of IPEC and etching of silica nanoparticles with HF leads to formation of various kinds of nano- and microcavities.

\section{Acknowledgements}

Financial support from the Science Committee of the Ministry of Education and Science of the Republic of Kazakhstan (Contract No. 201) is greatly acknowledged. Authors thank Dr. M. Hahn from Fraunhofer Institute of Applied Polymers (Germany) for preparation of PDMDAAC microgel and PhD student M. Karesoja from Laboratory of Polymer Chemistry, Helsinki University (Finland) for SEM measurement.

\section{References}

[1] Mark J. E.: Some general trends in the area of organicinorganic composites. In 'Hybrid Organic-Inorganic Composites' (eds.: Mark J. E., Lee C. Y-C., Bianconi P. A.) ACS Symposium Series, Washington, 1-4 (1995).

[2] Hay J. N., Shaw S. J.: Organic-inorganic hybrids The best of both worlds? Europhysics News, 34, 1-8 (2003).

[3] Saegusa T., Chujo Y.: Organic-inorganic polymer hybrids. Die Makromolekulare Chemie, Macromolecular Symposia, 64, 1-9 (1992).

[4] Giannelis E. P.: Polymer layered silicate nanocomposites. Advanced Materials, 8, 29-35 (1996).

[5] Giannelis E. P., Krishnamoorti R., Manias E.: Polymer-silicate nanocomposites: Model systems for confined polymers and polymer brushes. Advances in Polymer Science, 138, 107-147 (1999).

[6] Kickelbick G.: Concepts for the incorporation of inorganic blocks into organic polymers on a nanoscale. Progress in Polymer Science, 28, 83-114 (2003).

[7] Manne S., Aksay I. A.: Thin films and nanolaminates incorporating organic/inorganic interfaces. Current Opinion in Solid State and Material Science, 2, 358364 (1997).

[8] Harrup M. K., Wertsching A. K., Jones M. G.: Preparation and characterization of novel polymer/silicate nanocomposites. In: 'Functional Condensation Polymers' (eds.: Carraher C. E. Jr., Swift G. G.) Plenum Publishers, New York, 1-12 (2002).
[9] Cheng Y-J., Zhou S., Gutmann J. S.: Morphology transition in ultrathin titania films: From pores to lamellae. Macromolecular Rapid Communications, 28, 1392-1396 (2007).

[10] Avvaru N. R., de Tacconi N. R., Rajeshwar K.: Compositional analysis of organic-inorganic semiconductor composites. Analyst, 123, 113-116 (1998).

[11] Kurokawa Y., Sasaki M.: Complexation between polyions and hydrous inorganic oxides and adsorption properties of complex. Makromolekulare Chemie, 183, 679-685 (1982).

[12] Brinker C. J., Scherer G. W.: Sol-gel science. Academic, New York (1990).

[13] Kasaikin V. A., Pavlova N. V., Zezin A. B., Kabanov V. A.: Flocculation and stabilization of colloid silica by linear polyelectrolytes is the result of cooperative reactions between complementary particles (in Russian). Doklady Academii Nauk SSSR, 281, 888-892 (1985).

[14] Kasaikin V. A., Pavlova N. V., Ermakova L. N., Zezin A. B., Kabanov V. A.: Flocculation and stabilization of colloid silica by linear synthetic polyelectrolytes (in Russian). Journal of Colloids, 48, 452-460 (1986).

[15] Ermakova L. N., Alexandrova T. A., Nuss P. V., Wasserman A. M., Kasaikin V. A., Zezin A. B., Kabanov V. A.: Conformation of ionic macromolecules adsorbed on the surface of polysilica acid sols (in Russian). Polymer Science, Series A, 27, 18451851 (1985).

[16] Kalyuzhnaya R. I., Khulchaev Kh. Kh., Kasaikin V. A., Zezin A. B., Kabanov V. A.: Flocculation of polysilica acid sols by poly(dimethylaminoethylmethacrylate) (in Russian). Polymer Science, 36, 257-263 (1994).

[17] Kabanov V. A.: Polyelectrolyte complexes in solution and condensed phase (in Russian). Russian Review in Chemistry, 74, 3-20 (2005).

[18] Bixler H. J., Michaelis A. S.: Polyelectrolyte complexes. in 'Encyclopedia of Polymer Science and Technology' (eds.: Mark H. F., Gaylord N. G.) Interscience, New York, Vol. 10, 765-780 (1969).

[19] Tsuchida E., Abe K.: Interactions between macromolecules in solution and intermacromolecular complexes. Advances in Polymer Science, 45, 1-119 (1982).

[20] Zezin A. B., Kabanov V. A.: Novel class of complex water-soluble polyelectrolytes (in Russian). Russian Review in Chemistry, 51, 1447-1483 (1982).

[21] Bekturov E. A., Bimendina L. A.: Interpolymer complexes. Advances in Polymer Science, 41, 99-148 (1981).

[22] Philipp B., Dautzenberg H., Linow K-J., Kötz J., Dawydoff W.: Polyelectrolyte complexes - recent developments and open problems. Progress in Polymer Science, 14, 91-172 (1989).

[23] Ogawa K., Sato S., Kokufuta E.: Formation of intraand inetrparticle polyelectrolyte complexes between cationic nanogel and strong polyanion. Langmuir, 21, 4830-4836 (2005). 
[24] Khutoryanskaya O. V., Williams A. C., Khutoryanskiy V. V.: pH-mediated interactions between poly(acrylic acid) and methylcellulose in the formation of ultrathin multilayered hydrogels and spherical nanoparticles. Macromolecules, 40, 7707-7713 (2007).

[25] Donath E., Sukhrukov G., Caruso F., Davis S., Möhwald H.: Novel hollow polymer shells by colloid-templated assembly of polyelectrolytes. Angewandte Chemie, 37, 2201-2205 (1998).

[26] Hornok V., Erdőhelyi A., Dékány I.: Preparation of ultrathin membranes by layer-by-layer deposition of layered double hydroxide (LDH) and polystyrene sulfonate (PSS). Colloid and Polymer Science, 283, 1050-1055 (2005).

[27] Caruso F., Schüler C.: Enzyme multilayers on colloid particles: Assembly, stability, and enzymatic activity. Langmuir, 16, 9595-9603 (2000).

[28] Kotov N. A., Dékány I., Fendler J. H.: Layer-by-layer self-assembly of polyelectrolyte-semiconductor nanoparticle composite films. Journal of Physical Chemistry, 99, 13065-13069 (1995).

[29] Ai S., Lu G., He Q., Li J.: Highly flexible polyelectrolyte nanotubes. Journal of the American Chemical Society, 125, 11140-11141 (2003).
[30] Martinez Y., Retuert J., Yazdani-Pedram M., Cölfen H.: Hybrid ternary organic-inorganic films based on interpolymer complexes of silica. Polymer, 45, 3257 3265 (2004).

[31] Martinez Y., Retuert J., Yazdani-Pedram M., Cölfen H.: Transparent semiconductor-polymer hybrid films with tunable optical properties. Journal of Materials Chemistry, 17, 1094-1101 (2007).

[32] Koetz J., Kosmella S.: Polyelectrolytes and nanopartciles. Springer Verlag, Berlin (2007).

[33] Su D. T.: Gels formed from anionic and cationic polymers. US Patent 4501834, USA (1985).

[34] Chu B.: Laser light scattering: Basic principles and practice. Dover Publications Inc., New York (2007).

[35] Kratochvil P.: Classical light scattering from polymer solution. Elsevier, Amsterdam (1987).

[36] Feng Z., Wang Z., Gao C., Shen J.: Hollow microcapsules with a complex polyelectrolyte shell structure fabricated by polymerization of 4-vinylpyridine in the presence of poly(sodium 4-styrenesulfonate) and silica particles. Materials Letters, 61, 2560-2564 (2007).

[37] Rogacheva V. B., Grishina N. V., Zezin A. B., Kabanov V. A.: Intermacromolecular amidization in dilute aqueous solution of polyelectrolyte complex of polyacrylic acid and polyethylene imine (in Russian). Polymer Science, Series A, 25, 1530-1535 (1983). 